\title{
The Effect of Endometrial Polyps on Outcomes of In Vitro Fertilization (IVF) Cycles
}

\author{
A. LASS,${ }^{1,2}$ G. WILLIAMS, ${ }^{1}$ N. ABUSHEIKHA, ${ }^{1}$ and P. BRINSDEN ${ }^{1}$
}

Submitted: February 28, 1998

Accepted: March 29, 1999

Purpose: Our purpose was to investigate the effect of endometrial polyps on pregnancy outcome in an in vitro fertilization (IVF) program.

Methods: Endometrial polyps less than $2 \mathrm{~cm}$ in diameter were suspected by transvaginal ultrasound before oocyte recovery in 83 patients. Forty-nine women (Group I) had standard IVF-embryo transfer, while in 34 women (Group II) hysteroscopy and polypectomy were performed immediately following oocyte retrieval, the suitable embryos were all frozen, and the replacement cycle took place a few months later.

Results: Of the 32 hysteroscopies, a polyp was diagnosed in 24 cases (75\%) and polypoid endometrium in another 5 patients $(15.6 \%)$. An endometrial polyp was confirmed by histopathological examination in 14 women (58.3\%). The pregnancy rate in group I was similar to the general pregnancy rate of our unit over the same period (22.4 vs $23.4 \%)$ but the miscarriage rate was higher $(27.3$ vs $10.7 \%, P=$ 0.08 ). In Group II, the pregnancy and miscarriage rates were similar to those of the frozen embryo cycles at Bourn Hall (30.4 and 14.3 vs 22.3 and $12.1 \%$, respectively).

Conclusions: Small endometrial polyps, less than $2 \mathrm{~cm}$, do not decrease the pregnancy rate, but there is a trend toward increased pregnancy loss. A policy of oocyte retrieval, polypectomy, freezing the embryos, and replacing them in the future might increase the "take-home baby" rate.

KEY WORDS: endometrial polyp; frozen embryos; hysteroscopy; in vitro fertilization; vaginal ultrasound.

\section{INTRODUCTION}

Despite much progress in the earlier days of in vitro fertilization (IVF), such as ovulation induction, oocyte retrieval, and culture medium, the maximum conception rate still does not usually exceed $30 \%$ per embryo

\footnotetext{
' Bourn Hall Clinic, Bourn, Cambridge CB3 7TR, UK

${ }^{2}$ To whom correspondence should be addressed.
}

transfer and at least $90 \%$ of apparently normal embryos fail to implant (1). Structural pathology in the uterine cavity may play an important role in subfertility, implantation failure, or miscarriage. However, the role of the endometrial polyp(s) in reproductive failure is not clear and is probably underestimated. Surprisingly there is a lack of data regarding the effect of polyps on the implantation rate or pregnancy outcome in IVF. The incidental finding of an endometrial polyp in an IVF cycle presents a management dilemma to the medical team and their patients. The aim of this retrospective study was to evaluate (a) the pregnancy rate and outcome after embryo transfer without removal of the polyps and (b) the possible beneficial approach of performing hysteroscopy and polypectomy immediately following oocyte retrieval, freezing all embryos (FAE), and replacing them a few months later.

\section{MATERIALS AND METHODS}

\section{Subjects}

The data from 5736 oocyte recovery cycles for IVF performed at Bourn Hall Clinic during the period January 1991 to May 1997 were analyzed retrospectively. In 83 of these cycles (1.4\%), an endometrial polyp was suspected and recorded.

A polyp was diagnosed if an hyperechoic endometrial mass was found by transvaginal sonography. An Hitachi EUB 515 ultrasound scanner (Hitachi, Japan) with a $5-\mathrm{MHz}$ transvaginal transducer was used. Most of the scans were performed by the same author (G.W). Each polyp was measured in three planes-maximal longitudinal, anteroposterior, and transverse - and the average diameter was calculated. In a few other cycles in which polyps larger than $20 \mathrm{~mm}$ were identified, the cycles were abandoned pending further investigation and treatment; they are excluded from this study. 
Immediately after detection of an intrauterine polyp, either at the time of the "baseline" scan following gonadotropin-releasing hormone (GnRH) agonist treatment ( 40 cycles; $48.2 \%$ ) or during the ovulation induction monitoring period (43 cycles; $51.8 \%$ ). Patients had a detailed discussion with their doctor and were given the choice of (a) proceeding with the IVF cycle to embryo replacement (Group I) or (b) proceeding to oocyte recovery, hysteroscopy and polypectomy in the same procedure, followed by insemination of the oocytes and freezing of the resulting embryos with the intention of performing frozen embryo replacement (FER) in the future (Group II). We did not recommend embryo replacement in the cycle of the hysteroscopy and polypectomy because of the risk of jeopardizing implantation due to endometrial bleeding. The decision on which action line to embark on was made by the patients before oocyte recovery with advice from their doctor that there are currently no data available in the literature regarding the effect of the polyp(s) and therefore the "best" option is not clear.

\section{Ovulation Induction Protocol}

A GnRH agonist, either buserelin (Supercur; Shire Pharmaceuticals Ltd., East Anton, Andover, Hants, UK) or naferaline (Synarel; Searle, High Wycombe, UK). Follicle stimulating hormone (FSH), highly purified FSH, or recombinant human FSH (Metrodin, Metrodin HP, or Gonal-F; Serono Laboratories Ltd., Welwyn Garden City, UK) was used for follicular stimulation in all 83 cycles. The regimes for ovarian stimulation and cycle monitoring have been described previously $(2,3)$. Transvaginal ultrasound assessment of follicular growth, and endometrial thickness and measurement of estradiol $\left(E_{2}\right)$ levels were begun on stimulation day 8. Human chorionic gonadotropin (hCG; Profasi; Serono), 10,000 IU, was injected intramuscularly or subcutanously when there were at least three follicles $\geq 16 \mathrm{~mm}$ in average diameter. Transvaginal ultrasound-directed oocyte recovery under general anesthesia was performed $32-36 \mathrm{hr}$ after the hCG injection. In vitro culture and insemination were carried out according to our normal laboratory procedure (4). In all 49 Group I cycles, fertilization was achieved and up to three embryos were transferred to the uterus 48-52 hr after oocyte collection. Luteal-phase support was provided with progesterone suppositories, either Cyclogest (Shire Pharmaceuticals Ltd.), $400 \mathrm{mg}$ bd, or Utrogestan (Besins, Laboratory Besins, Iscovesco, Paris, France), $200 \mathrm{mg}$ tid, for 15 days.

\section{Hysteroscopy and D\&C}

The 34 women in Group II had an hysteroscopy immediately after their oocyte recovery. The cervix was dilated to Hegar 7 and a 6-mm rigid hysteroscope (Karl Storz Endoscopy America Inc., Culver City, CA) was inserted under direct vision. Saline solution was used as the distension medium. The entire uterine cavity was inspected for the presence of polyps, the instrument withdrawn, and the polyps removed by ovum forceps followed by endometrial curettage. The hysteroscope was inserted again to ensure complete removal of all polyps. All endometrial samples were fixed in buffered formaldehyde, sectioned, and stained in hematoxylin-eosin for histological examination.

\section{Cryopreservation of Embryos}

In Group II the oocytes of five patients failed to fertilize, and in a sixth the sole embryo was not suitable for cryopreservation. In 28 cycles, 155 embryos (mean $=5.5$, range $=1-15)$ at the pronucleate stage were cryopreserved in $1.5 \mathrm{M}$ propanediol (4).

\section{Frozen Embryo Replacement (FER)}

Of the 28 patients in Group II, 5 women, 2 of whom had only one embryo frozen, declined to have the embryos back for various personal reasons. Twentythree patients intended to start an FER cycle at least 3 months after oocyte retrieval. The embryos were replaced in either a "natural" monitored cycle or hormone-controlled cycles, as described previously by Sathanandan et al., (5).

\section{Pregnancy Monitoring}

Plasma samples were taken from the women 14 days after embryo transfer for measurement of hCG and progesterone concentrations. If hCG levels were $>10$ IU/L, then monitoring was continued at 5-day intervals. A transvaginal scan was performed about 35 days after embryo transfer to confirm the pregnancy. Clinical pregnancy was defined as the presence in the uterus of a fetus within a gestation sac, with evidence of fetal cardiac activity. Transient rises in hCG ("biochemical pregnancy") without evidence of a gestation sac, "blighted ova," or ectopic pregnancies were recorded but are not included in this analysis of data.

\section{Statistical Analysis}

The patients' age, fertility, obstetric history, total amount of FSH used, length of stimulation, peak $E_{2}$ 
levels, thickness of the endometrium, number of follicles aspirated, number of oocytes recovered, and number of embryos replaced were recorded. Fertilization and pregnancy rates were calculated. The data were statistically analyzed by the unpaired Student $t$-test and chi-square test where appropriate. A $P$ value of $<0.05$ was considered significant.

\section{RESULTS}

The two groups were similar in their age, obstetric history, and number of previous IVF attempts, as well as their response to superovulation (Table I). The mean average diameter of the polyps in Group II was bigger than in Group I $(11.8 \pm 3$ vs $9.5 \pm 3.2 \mathrm{~mm} ; P=$ 0.002 ), although none of the polyps was larger than $20 \mathrm{~mm}$. This difference might be a result of the shared decision of the patients and doctors to freeze all embryos (FAE) in these cases of larger polyps.

In five cycles in Group II (14.7\%), none of the oocytes fertilized, while in Group I fertilization was achieved in all 49 cycles; therefore, the mean fertilization rate was lower in Group II, although without statistical significance. The cause of this difference is not clear, because the decision to FAE was made before the injection of hCG. Hysteroscopy and curettage were performed immediately following oocyte recovery thus, the fertilization data were not known until much later and had no effect on the allocation of patients to the two groups.

Hysteroscopy confirmed the ultrasound findings in more than $90 \%$ of cases. It is often difficult to distinguish between a true polyp and polypoid endometrium by ultrasound, especially after superovulation, which causes a thick late-proliferative endometrium. In five procedures hysteroscopy revealed polypoid endometrium and in only three cases the hysteroscopy showed a normal endometrium. The prevalence of polyps diagnosed by hysteroscopy was similar whether they were detected in the baseline scan (78.6\%) or through monitoring of the superovulation phase (72.2\%; Table II). There were no other abnormalities such as fibroids or intrauterine adhesions.

In all 34 endometrial samples from Group II there was no evidence of endometritis, hyperplasia, or neoplasia. Of the 24 cases with polyps identified by hysteroscopy; endometrial polyps were confirmed by histopathology examination in 14 samples (58.3\%). A polyp was not found in the specimens from eight patients in whom the hysteroscopy excluded an endometrial polyp.

In Group I all cycles reached the embryo transfer stage. The implantation rate was $9.3 \%$ (12 embryos with a fetal heart of 129 embryos transferred). Eleven women achieved clinical pregnancies $(22.4 \%$ per oocyte retrieval and per embryo transfer) and eight delivered at term, including one set of twins. Three pregnancies miscarried in the first trimester $(27.2 \%)$. Over the same period, similar implantation rates $(12.7 \%)$ and clinical pregnancy and miscarriage rates (1055 pregnancies in 4493 embryo transfer cycles and 113 miscarriages; 23.4 and $10.7 \%$, respectively; $P=$ 0.08 ) were achieved at Bourn Hall Clinic (Table III).

In twenty three patients of Group II, (FER) cycles were attempted, but in two cases $(8.7 \%)$ the embryos failed to thaw (one embryo for one patient and five for the other). Seven women achieved clinical pregnancies

Table I. Demographic Data and Response to Superovulation in Women with a Suspected Polyp Who Continued to Fresh Embryo Transfer Compared with Women Who Froze All Embryos (FAE), with Later Embryo Transfert

\begin{tabular}{|c|c|c|}
\hline & $\begin{array}{c}\text { Group I } \\
\text { IVF-ET } \\
(N=49)\end{array}$ & $\begin{array}{c}\text { Group II } \\
\text { FAE } \\
(N=34)\end{array}$ \\
\hline $\begin{array}{l}\text { Age } \\
\text { Mean number of previous pregnancies (range) } \\
\text { Mean number of previous IVF attempts (range) } \\
\text { Mean average diameter (mm) of polyp (range)* } \\
\text { Polyp observed on the baseline vaginal scan (\%) } \\
\text { Total dose of FSH (Ampoules of } 75 \mathrm{U} \text { ) } \\
\text { Number of days of follicular stimulation } \\
\text { Peak } \mathrm{E}_{2} \text { level (pg/ml) } \\
\text { Endometrium thickness (mm) } \\
\text { Mean number of oocytes retrieved } \\
\text { Fertilization rate }(\%)\end{array}$ & $\begin{array}{c}35.4 \pm 3.8 \\
0.53(0-10) \\
1.1(0-6) \\
9.5 \pm 3.2(4.3-19.3) \\
30(49.2) \\
39.0 \pm 18 \\
9.9 \pm 1.4 \\
1373 \pm 768 \\
13.2 \pm 2.4 \\
9.9 \pm 5.1 \\
61.1\end{array}$ & $\begin{array}{c}35.4 \pm 3.6 \\
0.44(0-6) \\
0.8(0-4) \\
11.8 \pm 3.0(6.4-18.6) \\
14(41.1) \\
36.2 \pm 15.6 \\
9.8 \pm 1.8 \\
1507 \pm 1087 \\
13.2 \pm 3.0 \\
10.4 \pm 6.2 \\
55.8\end{array}$ \\
\hline
\end{tabular}

${ }^{a}$ All values are means $\pm \mathrm{SD}$.

$* P<0.002$. 
Table II. Hysteroscopy and D\&C Findings on the Day of Oocyte Retrieval for Women with a Suspected Polyp on Ultrasound

\begin{tabular}{|c|c|c|c|}
\hline & $\begin{array}{c}\text { Polyp on } \\
\text { baseline } \\
\text { scan }\end{array}$ & $\begin{array}{c}\text { No polyp } \\
\text { on } \\
\text { baseline } \\
\text { scan }\end{array}$ & Total \\
\hline No. of patients & 14 & 20 & 34 \\
\hline No. of hysteroscopies & 14 & $18^{a}$ & 32 \\
\hline \multicolumn{4}{|l|}{ Hysteroscopy findings (\%) } \\
\hline Polyp & $11(78.6)$ & $13(72.2)$ & $24(75.0)$ \\
\hline Polypoid endometrium & $2(14.3)$ & $3(16.7)$ & $5(15.6)$ \\
\hline Normal & $1(7.1)$ & $2(11.1)$ & $3(9.4)$ \\
\hline Polyp on D\&C $(\%)^{b}$ & $6(54.5)$ & $8(61.5)$ & $14(58.3)$ \\
\hline
\end{tabular}

"In two cases hysteroscopy failed for technical reasons.

${ }^{b}$ Percentages are from the number of patients with a diagnosis of a polyp on hysteroscopy.

(30.4\%; implantation rate $=16.7 \%)$, one woman miscarried $(14.3 \%)$, and six have delivered, including one healthy set of triplets (Table III). There was no difference in pregnancy rates between the natural monitored and the hormone-controlled ( $2 / 6$ vs $5 / 15$, respectively) cycles. These implantation, pregnancy, and miscarriage rates were similar to our results of 597 pregnancies including 72 miscarriages of 2674 FER cycles in the same period $(12.4,22.3$, and $12.1 \%$, respectively; $P=\mathrm{NS}$ ).

In total, five cycles resulted in biochemical pregnancies, four in Group I and one in the FAE cycles (8.1 vs $4.8 \%$, respectively; $P=$ NS). There were no ectopic pregnancies in this study.

\section{DISCUSSION}

Endometrial polyps are usually asymptomatic incidental findings in assisted reproduction technology cycles. The term polyp is a clinical rather than a pathological one, referring simply to a growth which is attached by a pedicle, and not indicative of any histological feature. Polyps can be made up of immature endometrium, in which case they respond only to the growth effect of estrogen, without any progesterone response (the most common type), or functional endometrium, which responds to ovarian hormones similarly to the general endometrium (6). Therefore, during the ovulation induction period, with high doses of estrogen as in IVF cycles, the thickened endometrium might mistakenly be considered a polyp or polypoid endometrium on transvaginal ultrasonography (7).

Recently, Kupfer et al. (8) demonstrated that transvaginal ultrasound is an accurate method to detect benign endometrial polyps, with a good correlation to hysteroscopic and histologic findings. Almost all of these masses were significantly less than $2 \mathrm{~cm}$ in size and were discrete foci. Furthermore, even small lesions, less than $1 \mathrm{~cm}$ in size, were easily identified. The best method of diagnosing polyps is by hysteroscopy $(9,10)$. Both late-proliferative and secretory endometrium may have an appearance similar to that of endometrial polyps, viewed by ultrasound and/or hysteroscopy. At hysteroscopy in the premenstrual phase the endometrium is often thrown into folds, which produces a polypoid appearance. This procedure is therefore usually performed in the postmenstrual phase of the cycle. In this study, on the contrary, the hysteroscopy was done after FSH superovulation for IVF in the presence of supraphysiological levels of estrogen and about $36 \mathrm{hr}$ after hCG injection, when the endometrium was relatively thick. It may be therefore that a few of the "polyps" were in fact genuine polypoid endometrium. In only three cases $(9.4 \%)$ was the conclusion of the hysteroscopist normal endometrium.

Endometrial polyps can be removed by blind curettage, but many are missed $(11,13)$, and therefore, they are more successfully treated with hysteroscopic guidance (11) or curettage followed by reintroducing the hysteroscope to ensure that all the lesions have been removed (13). The histological confirmation of a polyp in only $58 \%$ of cases when a polyp was confirmed by hysteroscopy might have resulted from misdiagnosis

Table III. Pregnancy Outcome in Cycles with a Suspected Polyp that Continued to Fresh Embryo Transfer (Group I) and FER Cycles Following FAE (Group II) Compared to the Total IVF Outcome at Bourn Hall Clinic (BHC) in the Same Period

\begin{tabular}{lcccc}
\hline & Group I & & Group II \\
FAE & FER at BHC \\
\hline $\begin{array}{l}\text { No. of cycles with embryos available for freezing } \\
\text { (FAE) or transfer (IVF-ET) }\end{array}$ & 49 & & & \\
Clinical pregnancies & $11(22.4 \%)$ & $1055(23.4 \%)$ & $7(33.3 \%)$ & $597(22.3 \%)$ \\
Implantation rate & $9.3 \%$ & $12.7 \%$ & $16.7 \%$ & $12.4 \%$ \\
Miscarriages (\% of clinical pregnancy) & $3(27.3 \%)$ & $113(10.7 \%)$ & $1(14.3 \%)$ & $72(12.1 \%)$ \\
\hline
\end{tabular}


of polypoid endometrium as a true polyp or from our method of blind curettage after hysteroscopy, especially with the smaller polyps.

Uterine anomalies, fibroids, intrauterine synechiae, and endometrial polyps are commonly considered to be closely associated with spontaneous and recurrent miscarriages, although there is still controversy about their incidence, classification, and role in reproductive failure (14) and, especially, in IVF treatment. Diagnostic hysteroscopy before IVF revealed intrauterine pathology (mainly adhesions) in up to $50 \%$ of patients (15-19) and a similar frequency in women who failed to conceive in one (17) or two (20) IVF cycles. Moreover, Dicker et al. (21) showed that in women with a previously normal hysteroscopy followed by three or more consecutive failed IVF-embryo transfer cycles, $18.2 \%$ demonstrated abnormalities on repeat hysteroscopy. Golan et al. (17) described a $22 \%$ pregnancy rate in IVF following surgical correction of intrauterine anomalies. In all these studies and others the abnormal uterine pathology findings were adhesions, septa, and fibroids, with very few endometrial polyps.

Endometrial polyps have been reported to occur in up to $24 \%$ of symptomatic patients (22), however, their incidence in the reproductive life span of the general population is not known. Taylor et al. (23) showed that the frequency of endometrial polyps in infertile women and women requesting reversal of sterilization (and, hence, probably fertile) was similar. We found endometrial polyps in $1.4 \%$ of patients who had IVF treatment. This is the first study, to our knowledge, that has investigated the effect of endometrial polyps on pregnancy outcome in an IVF program. The pregnancy rate in the group of patients that proceeded to fresh embryo transfer was similar to the overall figures at Bourn Hall Clinic. It seems that endometrial polyps smaller than $2 \mathrm{~cm}$ have no deleterious effect on conception following IVF treatment. The nearly three times higher miscarriage rate in Group I compared to our general IVF population (27.3 vs $10.3 \%$ ) was not significant, probably due to the relatively small number of patients. Power calculation shows that a minimum of 15 women conceiving with a polyp(s) with a similar miscarriage rate would be required to reach statistical significance of 0.05 .

In summary, we have shown that transvaginal ultrasound is a reliable method of detecting endometrial polyps before and through superovulation cycles for IVF. Small polyps, less than $2 \mathrm{~cm}$, do not decrease the implantation or pregnancy rate but may be a cause for increased pregnancy loss. We think that the presence of a small endometrial polyp before or through the stimulation for IVF should not alter the planned oocyte recovery and embryo transfer, however, further prospective and, preferably, multicentric collaborative studies are required to confirm these findings.

\section{ACKNOWLEDGMENTS}

We gratefully acknowledge Mrs. Margaret Hassouneh, SRN, for her invaluable assistance in collecting the pregnancy data.

\section{REFERENCES}

1. Human Fertilization and Embryology Authority: 5th Annual Report. HFEA, Paxton House, London, UK, 1996

2. Edwards RG, and Steptoe PC: Current status of human in vitro fertilization and implantation of human embryos. Lancet 1983;2:1265-1269

3. Macnamee $C$, Brinsden PR: Superovulation strategies in assisted conception. In A Textbook of In Vitro Fertilization and Assisted Reproduction, PR Brinsden, PA Rainsbury (eds). Parthenon, Carnforth, UK, 1992, pp 111-126

4. Elder KT, Aver SM: Routine gamete handling: Oocyte collection and embryo culture. In A Textbook of In Vitro Fertilization and Assisted Reproduction, PR Brinsden, PA Rainsbury (eds). Parthenon, Carnforth, UK, 1992, pp 155-170

5. Sathanandan M, Macnamee MC, Wick K, et al: Clinical aspects of human embryo cryopreservation. In A Textbook of In Vitro Fertilization and Assisted Reproduction, PR Brinsden, PA Rainsbury (eds). Parthenon, Carnforth, UK, 1992, pp 251 264

6. Jones HW: Endometrial polyps. In Novak's Textbook of Gynecology, 11th ed, HW Jones, AC Wentz, LS Burnett (eds). Williams \& Wilkins, Baltimore, MD, 1988, pp 723-727

7. Ayida G, Chamberlain P, Barlow D, Kennedy S: Uterine cavity assessment prior to in vitro fertilization: Comparison of transvaginal scanning, saline contrast hysterosonography and hysteroscopy. Ultrasound Obstet Gynecol 1997;10:59-62

8. Kupfer MC, Schiller VL, Hansen GC, Tessler FN: Transvaginal sonographic evaluation of endometrial polyps. J. Ultrasound Med 1994;13:535-539

9. Fedele L, Bianchi S, Dorta M, et al.: Transvaginal ultrasonography versus hysteroscopy in the diagnosis of uterine submucous myomas. Obstet Gynecol 1991;38:743-751

10. Campana A, de Agostini A, Bischof P, et al.: Evaluation of infertility. Hum Reprod Update 1995;1:586-606

11. Gimpelson R, and Rappold H: A comparative study between panoramic hysteroscopy with directed biopsies and curettage. A review of 276 cases. Am J Obstet Gynecol 1988;158:489-492

12. Loffer FD: Hysteroscopy with selective endometrial sampling compared with D\&C for abnormal uterine bleeding: the value of a negative hysteroscopic view. Obstet Gynecol 1989;73:16-20

13. Taylor PJ, Kredentser JV: Diagnostic and therapeutic laparoscopy and hysteroscopy and their relationship to in vitro fertiliza- 
tion. In A Textbook of In Vitro Fertilization and Assisted Reproduction, PR Brinsden, PA Rainsbury, (eds). Parthenon, Carnforth, UK, 1992, pp 73-92

14. Bulletti C, Flamigni C, Giacommucci E: Reproductive failure due to spontaneous abortion and recurrent miscarriage. Hum Reprod Update 1996;2:118-136

15. Seinera $P$, Maccario S, Visentin L, DiGregorio A: Hysteroscopy in an IVF-ER program. Acta Obstet Gynecol Scand 1988;67:135-137

16. Bordt J, Belkien L, Vancaillie $\mathrm{T}$, et al: Results of diagnostic hysteroscopies in an in vitro fertilization/embryo transfer program. Geburtshilfe Frauenheilkd 1984;44:813-815

17. Golan A, Ron-El R, Herman A, et al.: Diagnostic hysteroscopy: Its value in an in-vitro fertilization/embryo transfer unit. Hum Reprod 1992;7:1433-1434

18. Dicker D, Goldman JA, Ashkenazi J, et al: The value of hysteroscopy in elderly women prior to in vitro fertilization- embryo transfer (IVF-ET): A comparative study. J. Vitro Fert Embryo Transfer 1990;7:267-270

19. Shamma PN, Lee G, Gutmann JN, Lavy G: The role of hysteroscopy in in vitro fertilization. Fertil Steril 1992;58:1237-1239

20. Hamou J, Frydman R, Fernandez H: Evaluation prior to IVF by microhysterocopy. Abstracts of the VIth World Congress in IVF and Alternate Assisted Reproduction, Jerusalem, Israel, 1984, p 40

21. Dicker D, Ashkenazi J, Feldberg D, et al: : The value of repeat hysteroscopic evaluation in patients with failed in vitro fertilization transfer cycles. Fertil Steril 1992;58:833-835

22. VanBogaert $\mathrm{LJ}$ : Clinicopathologic findings in endometrial polyp. Obstet Gynecol 1988;71:771-773

23. Taylor PJ, Lewinthal D, Leader A, Pattinson AH: A comparison of dextran 70 with carbon dioxide as the distension medium for hysteroscopy in patients with infertility or requesting reversal of a prior tubal sterilization. Fertil Steril 1987:47:861-863 